\title{
Efficacy and Safety of Lenvatinib Therapy for Unresectable Hepatocellular Carcinoma in a Real-World Practice in Korea
}

\author{
Myung Ji Goh Joo Hyun Oh Yewan Park Jihye Kim Wonseok Kang \\ Dong Hyun Sinn Geum-Youn Gwak Yong-Han Paik Moon Seok Choi \\ Joon Hyeok Lee Kwang Cheol Koh Seung Woon Paik \\ Department of Medicine, Samsung Medical Center, Sungkyunkwan University School of Medicine, Seoul, \\ Republic of Korea
}

\section{Keywords}

Lenvatinib · Hepatocellular carcinoma · Efficacy · Safety ·

Real-world practice

\begin{abstract}
Background: Lenvatinib has been recently approved as a first-line treatment option for patients with unresectable hepatocellular carcinoma (HCC) in Korea. We aimed to study the efficacy and safety of lenvatinib therapy in a real-world practice and to find prognostic factors related to survival and disease progression. Methods: A hospital-based retrospective study was conducted on 111 consecutive patients who had unresectable HCC and were treated with lenvatinib at Samsung Medical Center from October 2018 to March 2020. Efficacy was determined using the modified Response Evaluation Criteria in Solid Tumors (mRECIST) criteria in 111 patients who completed 1st tumor assessment. Safety was evaluated in $116 \mathrm{HCC}$ patients including 5 patients who discontinued lenvatinib due to adverse events (AEs) before $1 \mathrm{st}$ tumor assessment using Common Terminology Criteria for AEs version 5.0. Results: A total of 111 patients with a median age of 59 years were analyzed during a median followup duration of 6.2 (4.4-9.0) months. The Kaplan-Meier esti-
\end{abstract}

karger@karger.com www.karger.com/lic

Karger $\stackrel{\text { ' }}{5}$

GOPEN ACCESS
(C) 2021 The Author(s)

Published by S. Karger AG, Basel

This article is licensed under the Creative Commons AttributionNonCommercial-NoDerivatives 4.0 International License (CC BYNC-ND) (http://www.karger.com/Services/OpenAccessLicense). Usage and distribution for commercial purposes as well as any distribution of modified material requires written permission. mate of overall survival was 10.5 months, and the median progression-free survival was 6.2 months. Based on mRECIST criteria, the objective response rate was $18.9 \%$ and disease control rate was $75.7 \%$. AEs developed in 86/116 (74.1\%) patients, and grade $\geq 3$ AEs developed in 16/116 (13.8\%) patients. Diarrhea, hand-foot skin rash, abdominal pain, hypertension, and anorexia were identified as the AEs with the highest frequencies of any grade. REFLECT eligibility criteria including tumor extent $\geq 50 \%$ liver occupation or inadequate bone marrow function and occurrence of anorexia were prognostic factors for survival, and occurrence of diarrhea was a favorable factor for disease progression. Conclusion: Lenvatinib therapy showed a favorable efficacy and safety in a real-world practice. The REFLECT eligibility criteria and specific AEs could be one of the prognostic markers.

(C) 2021 The Author(s)

Published by S. Karger AG, Basel

\section{Introduction}

Hepatocellular carcinoma (HCC) is the sixth most common cancer and the third leading cause of cancerrelated death worldwide [1]. Despite the advancements in imaging tests and therapeutic techniques during the last 
Fig. 1. Patient flow. HCC, hepatocellular carcinoma.

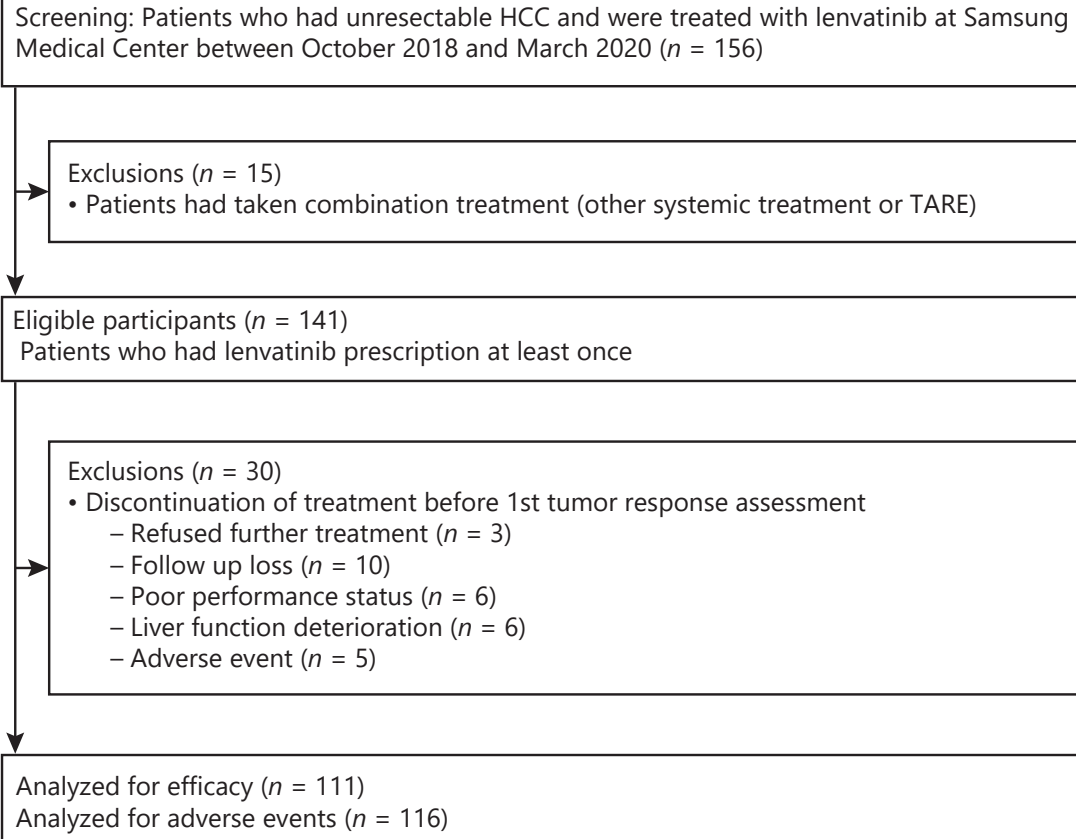

decade, the prognosis of advanced HCC is still poor that 5 -year survival rates are just $18 \%$ [2]. For patients with advanced stage and intermediate stage unsuitable for locoregional treatment, systemic treatment is the only therapeutic option [3].

Sorafenib, an oral multityrosine kinase inhibitor (TKI), has been the first-line treatment for advanced HCC since 2007, when the SHARP trial demonstrated that sorafenib improved median overall survival (OS) compared to placebo in patients with advanced HCC (10.7 vs. 7.9 months, $p<0.001$ ) [4]. For 10 years thereafter, numerous studies assessing new targeted agents as monotherapy including brivanib, sunitinib, and linifanib [5-7] or in combination with sorafenib including doxorubicin [8] and erlotinib [9] failed to demonstrate superiority or noninferiority compared to sorafenib in the first-line setting [10].

In 2018, lenvatinib, an oral TKI that inhibits VEGF receptors 1-3, FGF receptors 1-4, PDGF receptor $\alpha$, RET, and KIT [11], has been emerging as the first drug to show noninferiority compared to sorafenib in the first-line treatment for unresectable HCC patients [12]. The REFLECT trial, an open-label, phase 3, multicenter trial demonstrated that lenvatinib showed not only comparable OS with sorafenib (13.6 vs. 12.3 months) but also significant improvement in treatment response (overall re- sponse rate 40.6 vs. $12.4 \%)$ and median progression-free survival (PFS) duration (7.4 vs. 3.7 months) [12].

Accordingly, lenvatinib has been recently approved as the first-line treatment option for patients with unresectable HCC in Korea. We aimed to study the efficacy and safety of lenvatinib therapy in a real-world practice and to find prognostic factors related to survival and disease progression.

\section{Methods}

\section{Study Design and Patients}

This was a hospital-based retrospective cohort study. Patients who had unresectable HCC with diagnoses confirmed histologically or clinically in accordance with the regional HCC guideline $[13,14]$ and were treated with lenvatinib at least once at Samsung Medical Center from October 1, 2018, to March 31, 2020, were screened for potential inclusion in the study. Among the 156 eligible patients, patients were excluded if they had taken concomitant treatment with other systemic treatment or locoregional therapy $(n=15)$, discontinuation of treatment before 1st tumor response assessment due to refusal of further chemotherapy $(n=3)$, follow-up loss $(n=10)$, poor performance status $(n=6)$, liver function deterioration $(n=6)$, or adverse events (AEs) $(n=5)$. A total of 111 consecutive patients with unresectable HCC were analyzed for treatment efficacy. Safety was evaluated in 116 HCC patients including 5 patients who stopped lenvatinib due to AEs before the 1st tumor assessment (Fig. 1). 


\section{Data Collection and Definition of Variables}

We collected data on clinical characteristics (age, gender, etiology, performance status, baseline hypertension, or diabetes mellitus), laboratory findings (blood cell count, liver function test, tumor markers including alpha-fetoprotein, and protein induced by vitamin $\mathrm{K}$ absence or antagonist-II), and radiological findings (the number and size of intrahepatic lesions, macrovascular invasion, and extrahepatic lesions). We also collected data on stage of HCC (Barcelona Clinic Liver Cancer [BCLC] stage [15] and modified Union for International Cancer Control [mUICC] stage [13]) at the introduction time of lenvatinib and previous treatment including resection, transarterial chemoembolization, transarterial radioembolization, radiation therapy, and systemic treatment. Further information for systemic treatment including TKIs (sorafenib and regorafenib) or immunotherapy (pembrolizumab, nivolum$\mathrm{ab}$, atezolizumab or durvalumab) were collected. In addition, we collected information for liver function at baseline including Child-Pugh classification and modified ALBI (mALBI) grade. mALBI grade was calculated based on serum albumin and total bilirubin values using the following formula: $\left[\right.$ ALBI score $=\left(\log _{10}\right.$ bilirubin $(\mu \mathrm{mol} / \mathrm{L}) \times 0.66)+($ albumin $(\mathrm{g} / \mathrm{L}) \times-0.085)]$, and defined by the following 4 grades: $\leq-2.6=$ grade $1 ;>-2.60$ to $\leq$ $-2.27=$ grade $2 \mathrm{a} ;>-2.27$ to $\leq-1.39=$ grade $2 \mathrm{~b} ;>-1.39=$ grade 3 [16]. Lastly, we collected information for dose of lenvatinib during study period. Relative dose intensity for initial 8 weeks of therapy (8W-RDI) was calculated as the actual cumulative dose within the initial 8 weeks of starting lenvatinib treatment divided by the recommended dose [17]. Patients were scheduled to visit at weeks 6-12 for assessment of treatment response and safety using contrast-enhanced dynamic computed tomography $(\mathrm{CT})$ or magnetic resonance imaging (MRI), laboratory findings including tumor markers, and physical findings.

\section{Treatment Regimens}

Lenvatinib was administered orally for patients with unresectable HCC. The lenvatinib dose depended on the patients' weight: those who weighed $<60 \mathrm{~kg}$ were administered $8 \mathrm{mg}$ of lenvatinib daily, while those who weighed $\geq 60 \mathrm{~kg}$ were initially administered $12 \mathrm{mg}$ of lenvatinib daily. Initial dose reduction was made from 12 to $8 \mathrm{mg}$ in patients with poor oral intake, poor hepatic reserve function, or suspicious infection by clinician's own decision. During the study period, dose reduction or treatment interruption was also made in patients who developed AEs of at least grade 2 or AEs considered uncontrollable despite symptomatic management. Lenvatinib was discontinued when any unacceptable or serious AEs or clinical tumor progression was observed.

\section{Assessment of Treatment Response}

Initial treatment response was evaluated by contrast-enhanced dynamic CT or MRI at baseline and at 6-12 weeks after treatment initiation. Initial treatment response was assessed according to the modified Response Evaluation Criteria in Solid Tumors (mRECIST) [18]. Thereafter, radiological evaluation using CT or MRI was carried out every 1-3 months among the patients who did not show disease progression. We also assessed best overall response using mRECIST. Best overall response was defined as the best response recorded from the start of lenvatinib treatment until disease progression. Objective response rate (ORR) was defined as complete response (CR) and partial response (PR), and disease control rate (DCR) was defined as CR, PR, and stable disease (SD).
The efficacy of lenvatinib was further evaluated among patients who did or did not fulfill the REFLECT eligibility criteria (i.e., tumor extent $<50 \%$ liver occupation, no obvious invasion of the main portal vein or bile duct, no history of previous systemic treatment, adequate liver/bone marrow function, or no history of liver transplantation at the introduction time of lenvatinib). Definitions for adequate liver or bone marrow function were based on the REFLECT eligibility criteria.

\section{Safety Assessment}

Safety assessment included recording of performance status by the Eastern Cooperative Oncology Group (ECOG), vital signs, laboratory testing, urinalysis, and physical findings. Drug-related AEs were graded according to the Common Terminology Criteria for Adverse Events version 5.0. AEs were evaluated in 116 patients including 5 patients who discontinued lenvatinib due to AEs. For safety assessment, patients were evaluated at week 2 of lenvatinib therapy using laboratory tests and physical findings. Thereafter, safety assessment was performed with treatment response assessment every 1-3 months. As in the treatment response analysis, AEs were further evaluated stratified to whether patients fulfilled the REFLECT eligibility criteria or not.

\section{Factors Associated with Overall Survival and Disease}

Progression

We evaluated whether survival and disease progression were different among patients who did or did not fulfill the REFLECT eligibility criteria. Subgroup analysis was conducted based on each detailed criteria of REFLECT eligibility criteria. Furthermore, we evaluated the association between AEs and prognosis of HCC in patients treated with lenvatinib therapy. Likewise, subgroup analysis was conducted based on each AE. However, abdominal pain was not included in this analysis since it was difficult to distinguish cancer pain.

\section{Statistical Analysis}

Continuous variables were expressed as the median with interquartile range (IQR). The categorical variables were expressed as counts with percentages. The continuous variables were compared using Mann-Whitney $\mathrm{U}$ tests and the categorical variables using Fisher's exact test. The Kaplan-Meier method was used to estimate OS and PFS. To identify the factors associated with OS and disease progression, multivariable analysis was performed using the Cox proportional hazard model for variables with $p$ values of $<0.10$ in the univariable analysis. Components of REFLECT eligibility criteria and individual AEs were analyzed, respectively, to identify prognostic factors. Statistical analyses were performed using SPSS statistics 25.0 (IBM Crop., Armonk, NY, USA), and $p<0.05$ was considered statistically significant.

\section{Results}

\section{Patient Characteristics}

A total of 111 patients were analyzed for efficacy after exclusion, and their characteristics are shown in Table 1. The median patient age was 59 years (range, 49-67 years), and $96(86.5 \%)$ patients were male. Most patients $(94.6 \%)$ 
Table 1. Baseline characteristics of the patients

\begin{tabular}{|c|c|c|c|c|}
\hline & $\begin{array}{l}\text { Entire cohort } \\
(N=111)\end{array}$ & $\begin{array}{l}\text { Fulfill } \\
\text { REFLECT criteria } \\
(n=48)\end{array}$ & $\begin{array}{l}\text { Did not fulfill } \\
\text { REFLECT criteria } \\
(n=63)\end{array}$ & $p$ value \\
\hline Age, years & $59(49-67)$ & $57(49-65)$ & $60(48-68)$ & 0.48 \\
\hline Male & $96(86.5)$ & $41(85.4)$ & $55(87.3)$ & 0.79 \\
\hline ECOG PS $\geq 1$ & $24(21.6)$ & $10(20.8)$ & $14(22.2)$ & 1.00 \\
\hline Previous history of hypertension & $37(33.3)$ & $17(35.4)$ & $20(31.7)$ & 0.69 \\
\hline Previous history of diabetes & $21(18.9)$ & $13(27.1)$ & $8(12.7)$ & 0.09 \\
\hline Etiology (VH vs. non-VH) & $85: 26$ & $34: 14$ & $51: 12$ & 0.26 \\
\hline BCLC stage $\mathrm{C}$ & $105(94.6)$ & $45(93.8)$ & $60(95.2)$ & 1.00 \\
\hline mUICC stage (III:IV-A:IV-B) & 7:13:91 & $3: 6: 39$ & $4: 7: 52$ & 0.98 \\
\hline Intrahepatic lesions $(0: 1: 2: 3)$ & $19: 26: 11: 55$ & $6: 16: 5: 21$ & $13: 10: 6: 34$ & 0.16 \\
\hline Macrovascular invasion & $55(49.5)$ & $20(41.7)$ & $35(55.6)$ & 0.18 \\
\hline Extrahepatic lesion & $91(82.0)$ & $39(81.3)$ & $52(82.5)$ & 1.00 \\
\hline Previous systemic treatment & $15(13.5)$ & $0(0.00)$ & $15(23.8)$ & $<0.001$ \\
\hline Previous TKIs & 12 & 0 & 12 & \\
\hline Immune checkpoint inhibitors & 12 & 0 & 12 & \\
\hline Both & 9 & 0 & 9 & \\
\hline Initial dose reduction & $14(12.6)$ & $4(8.3)$ & $10(15.9)$ & 0.27 \\
\hline Dose modification & $46(41.4)$ & $19(39.6)$ & $27(42.9)$ & 0.85 \\
\hline 8W-RDI, \%, median (min, max) & $100(41.1-100)$ & $100(44-100)$ & $100(41.1-100)$ & 0.54 \\
\hline $\mathrm{ANC} \times 10^{3} / \mu \mathrm{L}$ & $3.6(2.5-4.5)$ & $3.2(2.5-4.6)$ & $3.7(2.4-4.5)$ & 0.93 \\
\hline Hemoglobin, g/dL & $13.6(12.3-15.0)$ & $13.8(12.5-15.5)$ & $13.5(12.3-14.6)$ & 0.24 \\
\hline Platelet count $\times 10^{3} / \mu \mathrm{L}$ & $150(111-204)$ & $155(115-206)$ & $143(97-204)$ & 0.46 \\
\hline Total bilirubin, mg/dL & $0.7(0.5-1.1)$ & $0.6(0.5-0.9)$ & $0.9(0.5-1.3)$ & 0.02 \\
\hline AST, U/L & $50.0(33.0-95.0)$ & $45.5(34.0-59.5)$ & $53.0(33.0-116.0)$ & 0.07 \\
\hline $\mathrm{ALT}, \mathrm{U} / \mathrm{L}$ & $33.0(22.0-60.0)$ & $32.5(22.0-52.3)$ & $33.0(23.0-75.0)$ & 0.22 \\
\hline ALP, U/L & $129(102-171)$ & $133(106-158)$ & $127(96-201)$ & 0.82 \\
\hline PT, INR & $1.04(0.99-1.12)$ & $1.03(0.97-1.08)$ & $1.06(1.01-1.17)$ & 0.02 \\
\hline Albumin, g/dL & $4.1(3.7-4.3)$ & $4.1(3.9-4.5)$ & $4.0(3.6-4.3)$ & 0.03 \\
\hline Child-Pugh score (5:6:7:8) & $84: 23: 3: 1$ & $41: 7: 0: 0$ & $43: 16: 3: 1$ & 0.13 \\
\hline Modified ALBI (grade 1:2a:2b:3) & $20: 32: 48: 11$ & $11: 18: 17: 2$ & 9:14:31:9 & 0.06 \\
\hline $\mathrm{AFP}, \mathrm{ng} / \mathrm{mL}$ & $386(11-5,309)$ & $163(12-1,392)$ & $548(9-13,220)$ & 0.40 \\
\hline PIVKA-II, mAU/mL & $562(41-13,421)$ & $200(91-3,400)$ & $865(46-42,143)$ & 0.08 \\
\hline Median follow-up duration & $6.2(4.4-9.0)$ & $7.2(4.5-9.6)$ & $5.7(4.2-8.9)$ & 0.01 \\
\hline
\end{tabular}

Data are presented as median (range) or $n(\%)$. ECOG PS, Eastern Cooperative Oncology Group performance status; VH, viral hepatitis; BCLC, Barcelona Clinic Liver Cancer; mUICC, modified Union for International Cancer Control; TKIs, tyrosine kinase inhibitors; $8 \mathrm{~W}$-RDI, relative dose intensity of lenvatinib within 8 weeks; ANC, absolute neutrophil count; AST, aspartate aminotransferase; ALT, alanine aminotransferase; PT, prothrombin time; INR, international normalized ratio; AFP, alpha-fetoprotein.

were diagnosed with BCLC stage C, and $91(82.0 \%)$ patients had extrahepatic lesions. The median follow-up duration was 6.2 months (range 4.4-9.9 months). Fifteen (13.5\%) patients had experienced previous systemic treatment including other oral TKIs, immunotherapy, or both (sorafenib $[n=12]$, regorafenib $[n=4]$, pembrolizumab $[n=2]$, nivolumab $[n=3]$, durvalumab $[n=5]$, and atezolizumab $[n=2])$. In $14(12.6 \%)$ patients with poor oral intake, poor liver function (Child-Pugh score B, total bilirubin $>3 \mathrm{mg} / \mathrm{dL}$, and aspartate/alanine transaminase $>5$ $\times$ upper limit of normal range), or fever/high C-reactive protein, initial dose modification from 12 to $8 \mathrm{mg}$ of lenvatinib was performed by clinician's own decision. Sixtythree (56.8\%) patients did not fulfill the REFLECT eligibility criteria due to tumor extent $\geq 50 \%$ liver occupation, main portal vein/bile duct invasion, previous systemic treatment, inadequate liver/bone marrow function, or history of liver transplantation (online suppl. Fig. 1; see www.karger.com/doi/10.1159/000512239) for all online suppl. material. There was no significant difference in 
Table 2. Clinical response of lenvatinib according to REFLECT eligibility criteria at initial tumor assessment

\begin{tabular}{lcccc}
\hline Response & Overall cohort & $\begin{array}{l}\text { Fulfilled } \\
\text { REFLECT } \\
\text { eligibility criteria }\end{array}$ & $\begin{array}{l}\text { Did not fulfill } \\
\text { REFLECT } \\
\text { eligibility criteria }\end{array}$ & $p$ value \\
\hline Complete response, $n(\%)$ & $1(0.9)$ & $0(0.0)$ & $1(1.6)$ \\
Partial response, $n(\%)$ & $20(18.0)$ & $10(20.8)$ & $10(15.9)$ \\
Stable disease, $n(\%)$ & $63(56.8)$ & $31(64.6)$ & $32(50.8)$ \\
Progressive disease, $n(\%)$ & $27(24.3)$ & $7(14.6)$ & $20(31.7)$ & $17.5 \%$ \\
Objective response rate & $18.9 \%$ & $20.8 \%$ & $68.3 \%$ & 0.81 \\
Disease control rate & $75.7 \%$ & $85.4 \%$ & & 0.045 \\
\hline \multicolumn{7}{c}{ Data are presented as $n(\%)}$. & & & \\
\hline
\end{tabular}

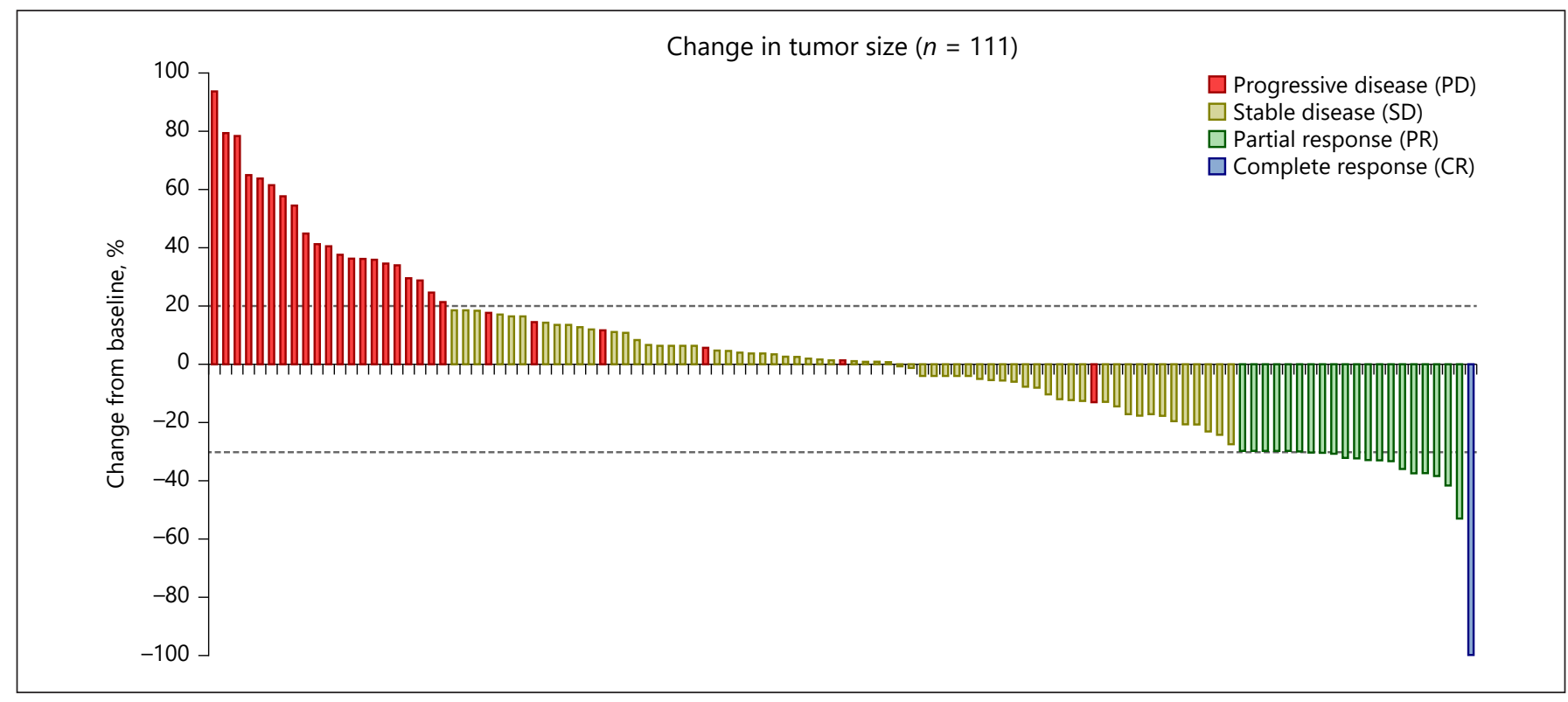

Fig. 2. Tumor reduction rate in the target lesion by mRECIST at the 1 st tumor assessment in 111 patients. CR/ PR was shown in 21 (18.9\%) patients, SD in 63 (56.8\%), and PD in 27 (24.3\%) patients, respectively. mRECIST, modified Response Evaluation Criteria in Solid Tumors; CR, complete response; PR, partial response; SD, stable disease; $\mathrm{PD}$, progressive disease.

baseline characteristics among patients who did or did not fulfill the REFLECT eligibility criteria except hepatic reserve function. Patients who did not fulfill the REFLECT eligibility criteria showed significantly higher portion of Child-Pugh score $\geq 6$ (14.6 vs. $31.7 \%$ ) and mALBI grade $\geq 2 b$ (39.5 vs. $63.5 \%)$.

\section{Treatment Response}

The first evaluation of the therapeutic efficacy of lenvatinib was performed at weeks $6-12$ of lenvatinib. Based on mRECIST criteria, CR/PR was shown in $21(18.9 \%)$ patients, SD in 63 (56.8\%), and PD in 27 (24.3\%) patients (ORR 18.9\%; DCR 75.7\%) (Fig. 2). Initial treatment response was the best overall treatment response during the study period. When comparing treatment response by mRECIST according to whether patients fulfilled the REFLECT eligibility criteria or not, there was no significant difference in ORR (20.8 vs. $17.5 \%, p$ value $=0.81)$ but there was significant difference in DCR (85.4 vs. $68.3 \%, p$ value $=0.045)($ Table 2). The Kaplan-Meier estimate of median OS was 10.5 months, and the median PFS was 6.2 months. 
Table 3. Adverse events $(N=116)$

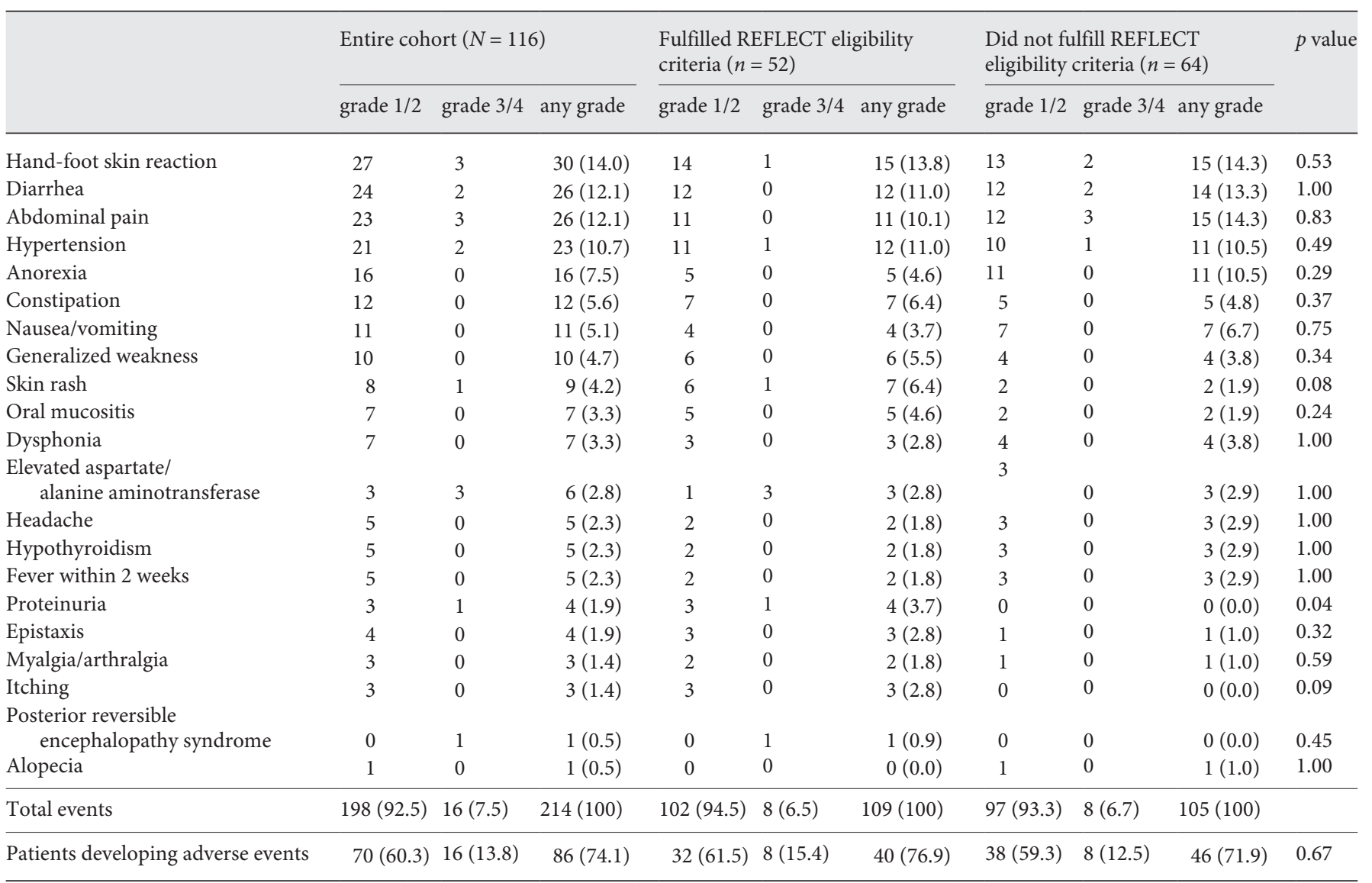

Data are presented as $n(\%)$.

\section{Safety Assessment}

A total of 214 AEs occurred in 86 of 116 (74.1\%) patients, and $\geq$ grade 3 AEs developed in 16 of $116(13.8 \%)$ patients (Table 3). Hand-foot skin reaction (HFSR), diarrhea, abdominal pain, hypertension, and anorexia were identified as the AEs with the highest frequencies of any grade. HFSR, diarrhea, abdominal pain, and elevated aspartate/alanine aminotransferase were identified as the AEs with the highest frequencies of grade 3 or 4 . Most AEs could be controlled by carrying out appropriate dose reduction, interruption, and symptomatic treatment. During the study period, dose reduction or interruption was performed in $35(31.5 \%)$ patients and 11 (9.9\%) patients discontinued lenvatinib due to cost $(n=1)$, deterioration of performance status $(n=2)$, recurrent hepatic encephalopathy $(n=4)$, intolerable abdominal pain $(n=$ $1)$, and infection $(n=3)$. There were $5(4.3 \%)$ patients who discontinued lenvatinib therapy before the first treatment response evaluation due to AEs of skin rash (grade 3), posterior reversible encephalopathy syndrome (grade 4), nausea/diarrhea/abdominal pain/hyperbilirubinemia (all grade 2), aspartate/alanine transaminase elevation (grade 3), and anorexia (grade 2) with aspartate/ alanine transaminase elevation (grade 3), respectively. Frequency of AEs was not different among patients who did or did not fulfill the REFLECT eligibility criteria (Table 3).

\section{Factors Associated with Overall Survival and Disease Progression}

When stratified according to whether patients fulfilled the REFLECT eligibility criteria or not, the Kaplan-Meier estimate of median OS was significantly different: 13.9 months (95\% confidence interval [CI]: 8.1-19.7) versus 8.7 months (95\% CI: 7.6-9.8), $p_{\log \text { rank }}=0.006$ (Fig. 3a). In univariable analysis, REFLECT criteria (i.e., tumor extent $\geq 50 \%$ of liver occupation, bile duct invasion, and inadequate bone marrow function), $\mathrm{mALBI}$ grade $\geq 2 \mathrm{~b}$, and an- 


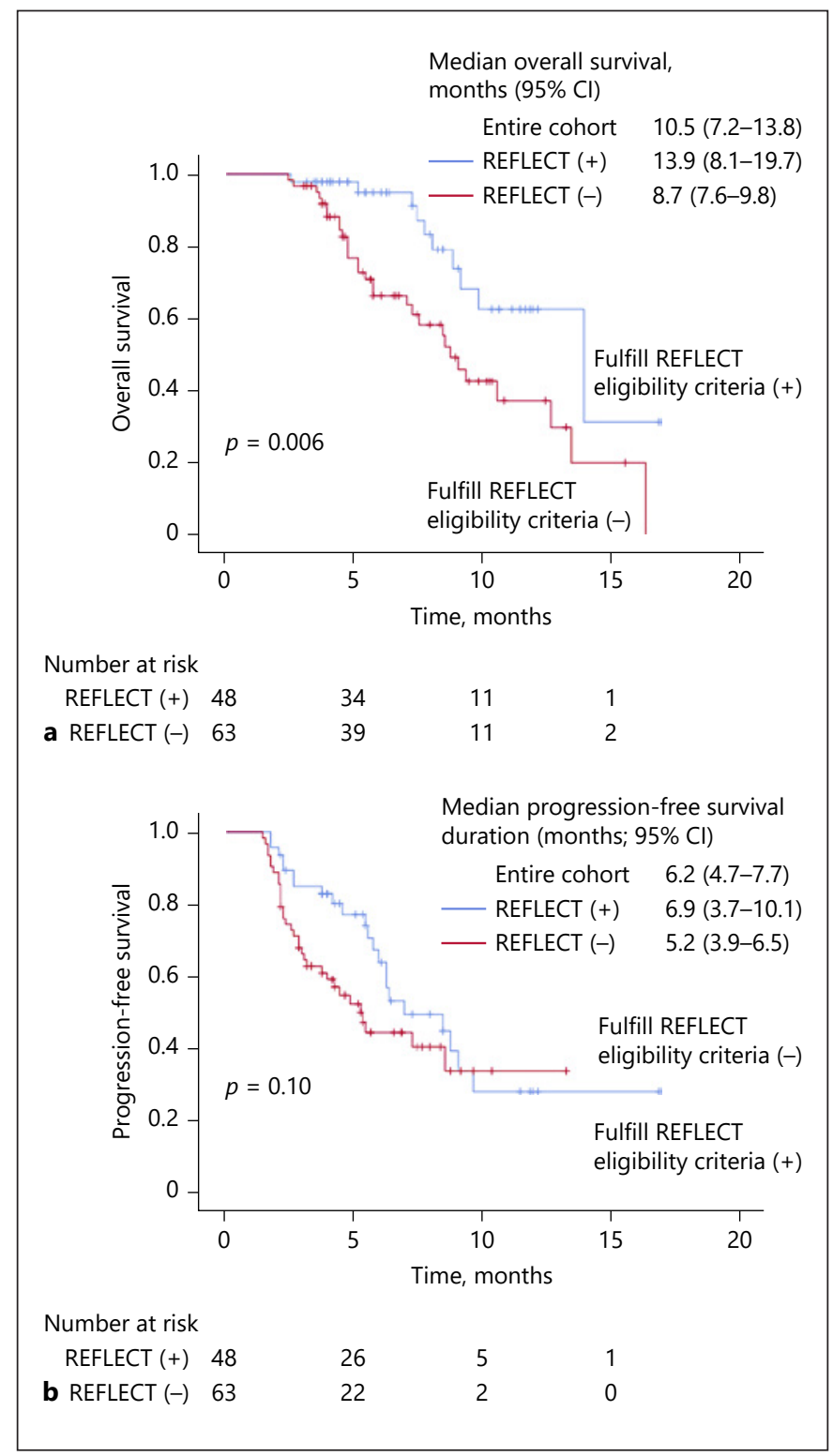

Fig. 3. Kaplan-Meier estimates of overall survival (a) and progression-free survival (b) according to whether patients fulfill the REFLECT eligibility criteria or not.

orexia of AEs were significantly associated with OS. In multivariable analysis, tumor extent $\geq 50 \%$ of liver occupation (adjusted hazard ratio [HR] $=4.37$; 95\% CI: 2.00 9.58) and inadequate bone marrow function $(\mathrm{HR}=3.77$; 95\% CI: 1.47-9.68) among REFLECT eligibility criteria and anorexia ( $\mathrm{HR}=2.15$; 95\% CI: 1.01-4.58) among AEs were found as poor prognostic factors for OS (Table 4).

However, whether patients fulfill the REFLECT eligibility criteria or not was not a significant prognostic fac- tor in PFS (6.9 months [95\% CI: 3.7-10.1] vs. 5.2 months [95\% CI: 3.9-6.5], $p_{\text {log rank }}=0.10$ ) (Fig. 3b). Tumor extent $\geq 50 \%$ of liver occupation was the only RELFECT eligibility criteria associated with poor PFS in univariable analysis. Meanwhile, specific AEs were significantly associated with PFS; occurrence of HFSR or diarrhea was associated with favorable PFS, and anorexia was associated with poor PFS. In multivariable analysis, diarrhea was found as the only favorable prognostic factor for disease progression during lenvatinib therapy $(\mathrm{HR}=0.37 ; 95 \% \mathrm{CI}$ : 0.17-0.84) (Table 5).

\section{Discussion}

In this study, lenvatinib showed a favorable efficacy and safety for patients with unresectable HCC in a realworld setting, though more than half of the patients $(n=$ $63,57.2 \%$ ) did not fulfill the REFLECT eligibility criteria. The ORR and DCR at initial treatment response at weeks 6-12 after starting lenvatinib was 18.9 and $75.7 \%$, which is consistent with the REFLECT trial (ORR: $24 \%$ and DCR: $75.5 \%$ by investigator review according to mRECIST) [12].

However, the current study found that patients who fulfilled the REFLECT eligibility criteria showed better DCR and OS than those of patients who did not fulfill the REFLECT eligibility criteria (DCR: 85.4 vs. $68.3 \%, p$ value 0.045 , median OS duration: 13.9 months [8.1-19.7] vs. 8.7 months [7.6-9.8]), which confronts the result from the recent Japanese study of 41 patients with unresectable HCC [19]. This is due to the difference in detail components of patients who did not fulfill the REFLECT eligibility criteria. In the previous study, patients did not fulfill the REFLECT eligibility criteria mainly due to history of previous systemic treatment $(16 / 23,69.6 \%)$ and all of them fulfill the REFLECT eligibility criteria of tumor extent. In contrast, about one-third (20/63, 31.7\%) had tumor extent $\geq 50 \%$ liver occupation in the current study. Among detail components of REFLECT eligibility criteria, tumor extent $\geq 50 \%$ liver occupation and inadequate bone marrow function were important prognostic factors for OS, but history of previous systemic treatment was not associated with OS or PFS in this study. There was no significant difference between patients who fulfilled the REFLECT eligibility criteria $(n=48)$ and patients who did not fulfill the REFLECT eligibility criteria only due to history of previous systemic treatment $(n=11)$ in OS and PFS (online suppl. Fig. 2) though the analysis is limited due to the small number of patients. 
Table 4. Multivariable Cox regression analysis for overall survival

\begin{tabular}{|c|c|c|c|c|}
\hline & \multicolumn{2}{|c|}{ Univariable analysis } & \multicolumn{2}{|c|}{ Multivariable analysis } \\
\hline & HR (95\% CI) & $p$ value & HR (95\% CI) & $p$ value \\
\hline Age $\geq 59$, median & $1.43(0.75-2.72)$ & 0.28 & & \\
\hline Male & $1.17(0.45-3.01)$ & 0.75 & & \\
\hline $\mathrm{ECOG} \geq 1$ & $1.55(0.78-3.12)$ & 0.22 & & \\
\hline Etiology (VH vs. non-VH) & $0.80(0.40-1.62)$ & 0.54 & & \\
\hline $\mathrm{mALBI} \geq 2 \mathrm{~b}$ & $2.02(1.05-3.87)$ & 0.03 & $1.31(0.63-2.74)$ & 0.48 \\
\hline mUICC (III, IV-A vs. IV-B) & $0.96(0.40-2.29)$ & 0.92 & & \\
\hline Intrahepatic lesion & $2.02(0.78-5.18)$ & 0.15 & & \\
\hline Extrahepatic involvement & $0.98(0.41-2.35)$ & 0.96 & & \\
\hline$\geq 50 \%$ liver volume & $3.11(1.52-6.33)$ & 0.002 & $4.37(2.00-9.58)$ & $<0.001$ \\
\hline Main portal vein involvement & $0.84(0.37-1.93)$ & 0.68 & & \\
\hline Bile duct involvement & $6.09(1.76-21.0)$ & 0.004 & $3.67(0.96-14.1)$ & 0.06 \\
\hline Previous systemic treatment & $0.79(0.33-1.89)$ & 0.59 & & \\
\hline Inadequate liver function & $2.31(0.80-6.68)$ & 0.12 & & \\
\hline Inadequate bone marrow function & $3.22(1.43-7.25)$ & 0.005 & $3.77(1.47-9.68)$ & 0.006 \\
\hline History of LT & $0.95(0.23-3.94)$ & 0.94 & & \\
\hline $8 \mathrm{~W}$-RDI <100\%, median & $1.53(0.71-3.27)$ & 0.28 & & \\
\hline AFP $\geq 386$, median & $1.58(0.84-2.98)$ & 0.16 & & \\
\hline PIVKA-II $\geq 562$, median & $1.46(0.78-2.74)$ & 0.24 & & \\
\hline HFSR & $0.75(0.36-1.59)$ & 0.45 & & \\
\hline Diarrhea & $0.57(0.25-1.30)$ & 0.18 & & \\
\hline Hypertension & $0.92(0.38-2.22)$ & 0.86 & & \\
\hline Anorexia & $2.55(1.27-5.13)$ & 0.009 & $2.15(1.01-4.58)$ & 0.05 \\
\hline History of hypertension & $1.81(0.94-3.45)$ & 0.08 & $1.78(0.87-3.64)$ & 0.11 \\
\hline History of diabetes & $1.43(0.68-3.01)$ & 0.35 & & \\
\hline
\end{tabular}

HR, hazard ratio; CI, confidence interval; ECOG, Eastern Cooperative Oncology Group; VH, viral hepatitis; mALBI, modified ALBI; LT, liver transplantation; 8W-RDI, relative dose intensity of lenvatinib within 8 weeks; AFP, alpha-fetoprotein; HFSR, hand-foot skin reaction.

Tumor burden and hepatic reserve function were the 2 most important factors in the prognosis of HCC [20, 21]. A previous multicenter study of 152 patients with unresectable HCC in Japan found that mALBI $\geq 2 b$ was the only prognostic factor for survival [22]. mALBI grade was also one of the prognostic factors in the univariable analysis in the current study but not in the multivariable analysis. This different result may be attributed to the difference in included patient characteristics. Patients with higher proportion of far advanced stage of HCC were included in the current study such as mUICC stage IV (93.7 vs. $51.3 \%$ ) and extrahepatic metastases ( 82.0 vs. $50 \%)$. Tumor burden could be more important factor than hepatic reserve function in patients with far advanced HCC.

However, we found that bone marrow function is one of the important prognostic factors for survival. Of 14 patients with inadequate bone marrow function, 1 patient did not meet hemoglobin $<8.5 \mathrm{~g} / \mathrm{dL}, 2$ patients did not meet $\mathrm{ANC}<1,500 / \mu \mathrm{L}$, and rest of them did not meet platelet $<75,000 / \mu \mathrm{L}$. This finding implicated that thrombocytopenia, which is the hallmark of advanced liver cirrhosis and portal hypertension [23], was associated with poor survival for advanced HCC. It is consistent with the result of recent meta-analysis that thrombocytopenia seems to be associated with poor OS and recurrence-free survival [24].

In the current study, $74.1 \%$ patients experienced at least one AE. The most common AEs of any grade were similar to those in the REFLECT trial, which included HFSR, diarrhea, and hypertension. However, proteinuria was developed in only 4 (1.9\%) patients in this study compared to $117 / 476$ (25\%) patients in the REFLECT trial. Grade $\geq 3$ AEs occurred in 16 of 116 (13.8\%) patients, which has much lower frequency than those in the previous studies $[12,25]$. In particular, there was only $2(1.7 \%)$ patients who need $\geq 2$ uncontrolled hypertension or SBP $>160$ during study period compared to $15-25 \%$ of patients developed hypertension $\geq$ grade 3 in previous stud- 
Table 5. Multivariable Cox regression analysis for progression-free survival

\begin{tabular}{|c|c|c|c|c|}
\hline & \multicolumn{2}{|c|}{ Univariable analysis } & \multicolumn{2}{|c|}{ Multivariable analysis } \\
\hline & $\operatorname{HR}(95 \% \mathrm{CI})$ & $p$ value & HR (95\% CI) & $p$ value \\
\hline Age $\geq 59$, median & $1.07(0.64-1.83)$ & 0.78 & & \\
\hline Male & $1.20(0.54-2.66)$ & 0.66 & & \\
\hline $\mathrm{ECOG} \geq 1$ & $1.75(0.96-3.20)$ & 0.07 & $1.81(0.96-3.40)$ & 0.07 \\
\hline Etiology (VH vs. non-VH) & $1.53(0.79-2.97)$ & 0.21 & & \\
\hline $\mathrm{mALBI} \geq 2 \mathrm{~b}$ & $1.06(0.62-1.81)$ & 0.83 & & \\
\hline mUICC (III, IV-A vs. IV-B) & $1.50(0.64-3.50)$ & 0.35 & & \\
\hline Intrahepatic lesion & $1.31(0.64-2.68)$ & 0.47 & & \\
\hline Extrahepatic involvement & $1.59(0.68-3.72)$ & 0.29 & & \\
\hline$\geq 50 \%$ liver volume & $2.01(1.02-3.96)$ & 0.04 & $1.46(0.72-2.94)$ & 0.29 \\
\hline Main portal vein involvement & $0.85(0.4-1.80)$ & 0.67 & & \\
\hline Bile duct involvement & $1.53(0.37-6.38)$ & 0.56 & & \\
\hline Previous systemic treatment & $1.46(0.73-2.93)$ & 0.28 & & \\
\hline Inadequate liver function & $1.36(0.49-3.877$ & 0.56 & & \\
\hline Inadequate bone marrow function & $1.40(0.63-3.13)$ & 0.41 & & \\
\hline History of liver transplantation & $1.44(0.45-4.65)$ & 0.54 & & \\
\hline $8 \mathrm{~W}-\mathrm{RDI}<100 \%$, median & $1.00(0.99-1.02)$ & 0.98 & & \\
\hline AFP $>386$, median & $1.16(0.69-1.98)$ & 0.57 & & \\
\hline PIVKA-II > 562, median & $1.16(0.68-1.97)$ & 0.59 & & \\
\hline HFSR & $0.38(0.19-078)$ & 0.008 & $0.51(0.24-1.08)$ & 0.08 \\
\hline Diarrhea & $0.30(0.13-0.66)$ & 0.003 & $0.37(0.17-0.84)$ & 0.02 \\
\hline Hypertension & $0.64(0.30-1.34)$ & 0.24 & & \\
\hline Anorexia & $1.83(0.96-3.47)$ & 0.07 & $1.51(0.78-2.94)$ & 0.22 \\
\hline History of hypertension & $1.36(0.78-2.37)$ & 0.27 & & \\
\hline History of diabetes & $1.51(0.81-2.82)$ & 0.20 & & \\
\hline
\end{tabular}

HR, hazard ratio; CI, confidence interval; ECOG, Eastern Cooperative Oncology Group; VH, viral hepatitis; mALBI, modified ALBI; 8W-RDI; relative dose intensity of lenvatinib within 8 weeks; AFP, alpha-fetoprotein; HFSR, hand-foot skin reaction.

ies $[12,25,26]$. Hence, compliance to lenvatinib therapy in these study patients was good, reflecting a median $8 \mathrm{~W}$ $\mathrm{RDI}$ of $100 \%$. There was no significant difference in $\mathrm{AE}$ occurrence rates and $8 \mathrm{~W}$-RDI among patients who did or did not fulfill the REFLECT eligibility criteria (Tables 1 , 3).

In addition, we investigated survival or disease progression according to each AE. The presence of HFSR has been regarded as a favorable factor of OS in patients treated with sorafenib [27] and regorafenib [28]. There were several studies that found specific AEs were associated with favorable outcome of lenvatinib therapy reflecting longer OS or time to progression (TTP) [22, 26, 29]; however, it is still inconclusive. In the current study, we found anorexia was the only factor associated with poor OS $(\mathrm{HR}=2.15,95 \% \mathrm{CI}: 1.01,4.58)$ after adjustment for factors related to tumor burden and hepatic reserve function. Moreover, we found that diarrhea and HFSR have the potential to predict longer TTP during lenvatinib therapy. Diarrhea was the only favorable prognostic factor for disease progression in multivariable analysis (HR $=0.37 ; 95 \%$ CI: $0.17-0.84, p$ value $=0.02$ ), while HFSR was only marginally associated ( $\mathrm{HR}=0.51 ; 95 \% \mathrm{CI}$ : $0.24-$ $1.08, p$ value $=0.08$ ).

Of note, there were $23(20.7 \%)$ patients who received palliative radiation therapy within 1 month or during lenvatinib therapy. Palliative radiation therapy was performed to portal vein/hepatic vein tumor thrombus ( $n=$ $4)$, bone metastasis $(n=8)$, lymph node $(n=3)$, peritoneal seeding nodule $(n=3)$, or main liver lesion $(n=5)$. There was no dose modification of lenvatinib for considering additive AEs due to radiation therapy. However, radiation therapy within 1 month or during lenvatinib therapy was not significantly aggravated developing AEs of any grade. Efficacy evaluated by initial treatment response at weeks 6-12 during lenvatinib was higher in pa-
60

Liver Cancer 2021;10:52-62

DOI: $10.1159 / 000512239$
Goh/Oh/Park/Kim/Kang/Sinn/Gwak/ Paik/Choi/Lee/Koh/Paik 
tients who received concurrent radiation therapy; however, ORR (18.2\%) and DCR (73.9\%) was still favorable after excluding patients who received radiation therapy (online suppl. Table 1). Receiving radiation therapy within 1 month or during lenvatinib therapy was not significantly associated with OS or disease progression (data not shown).

The strength of our study is a relatively homogenous cohort, in which the patients included in this study were mostly consisted of BCLC stage C/mUICC stage IV HCC with extrahepatic metastases. However, there are several limitations. First of all, this study was a retrospective study from a single tertiary center of a single ethnicity. Moreover, the number of cases and observation period were limited. Nevertheless, we found that tumor extent $\geq 50 \%$ liver volume and anorexia were poor prognostic factors for survival in patients with far advanced HCC and diarrhea among AEs was favorable prognostic factors for disease progression during lenvatinib therapy. This finding would be helpful to predict outcome of patients who were treated with lenvatinib therapy in the realworld clinical practice.

In conclusion, lenvatinib therapy showed a favorable efficacy and safety in a real-world practice, even though patients who did not meet the REFLECT eligibility criteria were included in this study. In addition, whether patients fulfilled the REFLECT eligibility criteria or not and the presence of specific AEs (anorexia and diarrhea) could be the prognostic factors associated with survival and disease progression during lenvatinib therapy.

\section{Statement of Ethics}

This study was performed in accordance with the Declaration of Helsinki, and it has been approved by the Institutional Review Board at the Samsung Medical Center (IRB No. 2020-03-119-001). As the study used only deidentified data routinely collected during hospital visits, the requirement of obtaining informed consent from the patients was waived.

\section{Conflict of Interest Statement}

The authors have no conflicts of interest to declare.

\section{Funding Sources}

This work was supported by the National Research Foundation of Korea (NRF) grant funded by the Korea government (MSIT) (NRF-2016R1C1B2015463 and NRF-2019R1C1C1007729).

\section{Author Contributions}

M.J.G. and W.K.: study design, statistical analysis, and drafting of the manuscript. J.H.O., Y.P., and J.K.: data interpretation and critical revision of the manuscript. D.H.S., G.Y.G., and M.S.C.: study design, study supervision, and critical revision of the manuscript. Y.H.P.: study design, drafting of the manuscript, and study supervision. J.H.L.: study supervision, statistical analysis, and data interpretation. K.C.K.: study design, study supervision, and data interpretation. S.W.P.: study supervision, data interpretation, and critical revision of the manuscript. All authors approved the final submission.

\section{References}

1 Siegel RL, Miller KD, Jemal A. Cancer statistics, 2016. CA Cancer J Clin. 2016 Jan-Feb; 66(1):7-30.

2 Li D, Sedano S, Allen R, Gong J, Cho M, Sharma S. Current treatment landscape for advanced hepatocellular carcinoma: patient outcomes and the impact on quality of life. Cancers. 2019 Jun 18;11(6):841.

3 Ogasawara S, Ooka Y, Koroki K, Maruta S, Kanzaki H, Kanayama K, et al. Switching to systemic therapy after locoregional treatment failure: definition and best timing. Clin Mol Hepatol. 2020 Apr;26(2):155-62.

4 Llovet JM, Ricci S, Mazzaferro V, Hilgard P, Gane E, Blanc JF, et al. Sorafenib in advanced hepatocellular carcinoma. N Engl J Med. 2008 Jul 24;359(4):378-90

5 Johnson PJ, Qin S, Park JW, Poon RT, Raoul JL, Philip PA, et al. Brivanib versus sorafenib as first-line therapy in patients with unresectable, advanced hepatocellular carcinoma: results from the randomized phase III BRISK-
FL study. J Clin Oncol. 2013 Oct 1;31(28): 3517-24.

6 Cheng AL, Kang YK, Lin DY, Park JW, Kudo $M$, Qin S, et al. Sunitinib versus sorafenib in advanced hepatocellular cancer: results of a randomized phase III trial. J Clin Oncol. 2013 Nov 10;31(32):4067-75.

7 Cainap C, Qin S, Huang WT, Chung IJ, Pan $\mathrm{H}$, Cheng $\mathrm{Y}$, et al. Linifanib versus sorafenib in patients with advanced hepatocellular carcinoma: results of a randomized phase III trial. J Clin Oncol. 2015 Jan 10;33(2): $172-9$.

8 Abou-Alfa GK, Shi Q, Knox JJ, Kaubisch A, Niedzwiecki D, Posey J, et al. Assessment of treatment with sorafenib plus doxorubicin vs sorafenib alone in patients with advanced hepatocellular carcinoma: phase 3 CALGB 80802 randomized clinical trial. JAMA Oncol. 2019 Sep 5;5(11):1582-8.

9 Zhu AX, Rosmorduc O, Evans TR, Ross PJ, Santoro A, Carrilho FJ, et al. SEARCH: a phase III, randomized, double-blind, placebo-controlled trial of sorafenib plus erlotinib in patients with advanced hepatocellular carcinoma. J Clin Oncol. 2015 Feb 20;33(6):55966.

10 Dika IE, Abou-Alfa GK. Treatment options after sorafenib failure in patients with hepatocellular carcinoma. Clin Mol Hepatol. 2017 Dec;23(4):273-9.

11 Yamamoto Y, Matsui J, Matsushima T, Obaishi $\mathrm{H}$, Miyazaki K, Nakamura K, et al. Lenvatinib, an angiogenesis inhibitor targeting VEGFR/FGFR, shows broad antitumor activity in human tumor xenograft models associated with microvessel density and pericyte coverage. Vasc Cell. 2014;6:18.

12 Kudo M, Finn RS, Qin S, Han KH, Ikeda K, Piscaglia F, et al. Lenvatinib versus sorafenib in first-line treatment of patients with unresectable hepatocellular carcinoma: a randomised phase 3 non-inferiority trial. Lancet. 2018 Mar 24;391(10126):1163-73.
Efficacy and Safety of Lenvatinib for Unresectable HCC
Liver Cancer 2021;10:52-62

DOI: $10.1159 / 000512239$ 
13 Korean Liver Cancer Study Group (KLCSG) and National Cancer Center, Korea (NCC). 2014 KLCSG-NCC Korea Practice Guideline for the management of hepatocellular carcinoma. Gut Liver. 2015 May 23;9(3):267-317.

14 Kim TH, Kim SY, Tang A, Lee JM. Comparison of international guidelines for noninvasive diagnosis of hepatocellular carcinoma: 2018 update. Clin Mol Hepatol. 2019 Sep; 25(3):245-63.

15 Bruix J, Sherman M. Management of hepatocellular carcinoma: an update. Hepatology. 2011 Mar;53(3):1020-2.

16 Hiraoka A, Michitaka K, Kumada T, Izumi N, Kadoya M, Kokudo N, et al. Validation and potential of albumin-bilirubin grade and prognostication in a nationwide survey of 46,681 hepatocellular carcinoma patients in Japan: the need for a more detailed evaluation of hepatic function. Liver Cancer. 2017 Nov; 6(4):325-36.

17 Sasaki R, Fukushima M, Haraguchi M, Miuma S, Miyaaki H, Hidaka M, et al. Response to lenvatinib is associated with optimal relativeDose intensity in hepatocellular carcinoma: experience in clinical settings. Cancers. 2019 Nov 10;11(11):1769.

18 Lencioni R, Llovet JM. Modified RECIST (mRECIST) assessment for hepatocellular carcinoma. Semin Liver Dis. 2010 Feb;30(1): $52-60$.
19 Sho T, Suda G, Ogawa K, Kimura M, Shimazaki T, Maehara O, et al. Early response and safety of lenvatinib for patients with advanced hepatocellular carcinoma in a real-world setting. JGH Open. 2020 Feb;4(1):54-60.

20 A new prognostic system for hepatocellular carcinoma: a retrospective study of $435 \mathrm{pa}$ tients: the cancer of the Liver Italian Program (CLIP) investigators. Hepatology. 1998 Sep; 28(3):751-5.

21 Kudo M, Chung H, Haji S, Osaki Y, Oka H, Seki T, et al. Validation of a new prognostic staging system for hepatocellular carcinoma: the JIS score compared with the CLIP score. Hepatology. 2004 Dec;40(6):1396-405.

22 Hiraoka A, Kumada T, Atsukawa M, Hirooka M, Tsuji K, Ishikawa T, et al. Prognostic factor of lenvatinib for unresectable hepatocellular carcinoma in real-world conditions-multicenter analysis. Cancer Med. 2019;8(8):371928.

23 Peck-Radosavljevic M. Thrombocytopenia in liver disease. Can J Gastroenterol. 2000 Nov; 14(Suppl D):60D-6D.

24 Pang Q, Qu K, Bi JB, Liu SS, Zhang JY, Song $\mathrm{SD}$, et al. Thrombocytopenia for prediction of hepatocellular carcinoma recurrence: systematic review and meta-analysis. World J Gastroenterol. 2015;21(25):7895-906.
25 Ikeda K, Kudo M, Kawazoe S, Osaki Y, Ikeda $\mathrm{M}$, Okusaka T, et al. Phase 2 study of lenvatinib in patients with advanced hepatocellular carcinoma. J Gastroenterol. 2017 Apr;52(4): 512-9.

26 Shimose S, Iwamoto H, Niizeki T, Shirono T, Noda Y, Kamachi N, et al. Clinical significance of adverse events for patients with unresectable hepatocellular carcinoma treated with lenvatinib: a multicenter retrospective study. Cancers. 2020 Jul 11;12(7):1867.

27 Wang P, Tan G, Zhu M, Li W, Zhai B, Sun X. Hand-foot skin reaction is a beneficial indicator of sorafenib therapy for patients with hepatocellular carcinoma: a systemic review and meta-analysis. Expert Rev Gastroenterol Hepatol. 2018 Jan;12(1):1-8.

28 Bruix J, Merle P, Granito A, Huang Y-H, Bodoky G, Yokosuka O, et al. Hand-foot skin reaction (HFSR) and overall survival (OS) in the phase 3 RESORCE trial of regorafenib for treatment of hepatocellular carcinoma (HCC) progressing on sorafenib. J Clin Oncol. 2018; 36(4_suppl):412.

29 Sung MW, Finn RS, Qin S, Han K-H, Ikeda K, Cheng A-L, et al. Association between overall survival and adverse events with lenvatinib treatment in patients with hepatocellular carcinoma (REFLECT). J Clin Oncol. 2019;37(4_ suppl):317. 